\title{
Programming JADE and Jason agents based on social relationships using a uniform approach
}

\author{
Matteo Baldoni, Cristina Baroglio, Federico Capuzzimati \\ Università degli Studi di Torino - Dipartimento di Informatica \\ c.so Svizzera 185, I-10149 Torino (Italy) \\ \{matteo.baldoni, cristina.baroglio, federico.capuzzimati\}@unito.it
}

\begin{abstract}
Interaction is an essential feature in multiagent systems. Design primitives are needed to explicitly model desired patterns. This work presents 2COMM as a framework for defining social relations among parties, represented by social commitments. Starting from the definition of interaction protocols, 2COMM allows to decouple interaction design from agent design. Currently, adapters were developed for allowing the use of $2 \mathrm{COMM}$ with the JADE and the JaCaMo platforms. We show how agents for the two platforms can be implemented by relying on a common programming schema.
\end{abstract}

Keywords: Social Commitments · Agents \& Artifacts · Agent-Oriented Software Engineering

\section{Introduction and Motivation}

Multiagent Systems (MAS) are a preferred choice for building complex systems where the autonomy of each component is a major requirement. Agent-oriented software engineers can choose from a substantial number of agent platforms [18,11,16,5]. Tools like JADE [8], TuCSoN [20], DESIRE [12], JaCaMo [10], all provide coordination mechanisms and communication infrastructures [11] but, in our opinion, they lack of abstractions that allow a clear and explicit modeling of interaction. Basically, the way in which agents interact is spread across and "hard-coded" into agent implementations. This choice overly ties agent implementations with a negative impact on software reuse. A clear separation of the agents from the specification of their coordination would bring advantages both on the design and on the implementation of MAS by bringing in a greater decoupling.

To this purpose, we propose to explicitly represent agent coordination patterns in terms of normatively defined social relationships, and to ground this normative characterization on commitments [23] and on commitment-based interaction protocols [25]. Practically, we exploit 2COMM [1], a tool that allows building artifacts that incorporate commitment-based protocols, in a way that is not bounded to an agent platform, and two connectors that allow using 2COMM for making JADE agents and JaCaMo agents interact. 
Relying on artifacts has the advantage of transforming social relationships and coordination patterns into resources and this allows agents to dynamically recognize, accept, manipulate, reason on them, and decide whether to conform to them (a basis for coordination [15]). In order to reify the social relationships we rely on the Agents \& Artifacts meta-model (A\&A) [24,19], which provides abstractions for environments and artifacts, that can be acted upon, observed, perceived, notified, and so on. 2COMM adopts the abstraction of artifact to construct communication protocols that realize a form of mediated, programmable communication, and in particular commitment protocols to establish an interaction social state agents can use to take decisions about their behaviour. Through 2COMM protocol artifacts, social relationships can be examined by the agents, as advised in [14], used (which entails that agents accept the corresponding regulations), constructed, e.g., by negotiation, specialized, composed, and so forth. Finally, 2COMM artifacts enable the implementation of monitoring functionalities for verifying that the on-going interactions respect the commitments and for detecting violations and violators.

This work also shows how starting from interactions in building a system can be useful when programming socially-responsive agents. A clear specification of the commitments that an agent has to handle constitutes an outline, for agent implementation, that developers can follow.

Summarizing, this work proposes to introduce in MAS an explicit notion of social relationship, based on that of commitment (Section 2). The framework 2COMM (Section 3), an extension of JaCaMo, realizes the proposal. We explain programming schemas for JADE and Jason agents. We show the impact of the proposal on programming by means of an example (Section 4) based on the FIPA Contract Net Protocol (CNP). The example shows 1) practical advantages in terms of better code organization and easier coding of agents interaction, and 2 ) how agent implementation is lead by the interaction pattern, providing a cross-platform programming pattern.

\section{Modeling Social Relationships}

We propose to explicitly represent social relationships among the agents. By social relationships we mean normatively defined relationships, between two or more agents, resulting from the enactment of roles, and subject to social control. Thus, we encode social relationships as commitments. A commitment [22] is represented with the notation $\mathrm{C}(x, y, r, p)$, capturing that the agent $x$ commits to the agent $y$ to bring about the consequent condition $p$ when the antecedent condition $r$ holds. Antecedent and consequent conditions generally are conjunctions or disjunctions of events and commitments. When $r$ equals $T$, we use the short notation $\mathrm{C}(x, y, p)$ and the commitment is said to be active. Commitments have a regulative nature, in that debtors are expected to behave so as to satisfy the engagements they have taken. This practically means that an agent is expected to behave so as to achieve the consequent conditions of the active commitments of which it is the debtor. 
We envisage both agents and social relationships as first-class entities that interact in a bi-directional manner. Social relationships are created by the execution of interaction protocols and provide expectations on the agents' behaviour. It is, therefore, necessary to provide the agents the means to create, to manipulate, to observe, to monitor, to reason, and to deliberate on social relationships. We do so by exploiting properly defined artifacts, that reify both interaction protocols, defined in terms of social relationships, and the sets of social relationships, that are created during the protocols execution, available to agents as resources.

An artifact (A\&A meta-model $[24,19])$ is a computational, programmable system resource, that can be manipulated by agents, residing at the same abstraction level of the agent abstraction class. For their very nature, artifacts can encode a mediated, programmable and observable means of communication and coordination between agents. We interpret the fact that an agent uses an artifact as its explicit acceptance, of the implications of the interaction protocol that the artifact reifies. This allows the interacting parties to perform practical reasoning, based on expectations: a debtors of a commitment is expected to behave so as to satisfy the commitment consequent conditions; otherwise, a violation is raised.

A commitment-based protocol consists of a set of actions, whose semantics is shared, and agreed upon, by all of the participants to the interaction $[25,13]$. The semantics of the social actions is given in terms of commitment operations (as usual for commitments, create, cancel, release, discharge, assign, and delegate). The execution of commitment operations modifies the social state of the system, which is shared by the interacting agents. As in [22], we postulate that discharge is performed concurrently with the actions that lead to the given condition being satisfied and causes the commitment to not hold. Delegate and assign transfer commitments respectively to a different debtor and to a different creditor $[22,25,13]$. Commitment-based protocols provide a means of coordination, based on the notification of social events, e.g. the creation of a commitment. Agents use artifacts to coordinate and interact in a way that depends on the roles they play and on their objectives.

From an organizational perspective, a protocol is structured into a set of roles. We assume that roles cannot live autonomously: they exist in the system in view of the interaction. We follow the ontological model for roles proposed in [9], and brought inside the object-oriented paradigm in [6,7], which is characterized by three aspects: (1) Foundation: a role must always be associated with the institution it belongs to and with its player; (2) Definitional dependence: the definition of the role must be given inside the definition of the institution it belongs to; (3) Institutional empowerment: the actions defined for the role in the definition of the institution have access to the state of the institution and of the other roles, thus, they are called powers; instead, the actions that a player must offer for playing a role are called requirements. The agents that will be the role players become able to perform protocol actions, that are powers offered by a specific role and whose execution affect the social state. On the other hand, they need to satisfy the related requirements: specifically, in order to play a role 
an agent needs to have the capabilities of satisfying the related commitments capabilities which can be internal of the agent or supplied as powers as well.

\section{2COMM: a Commitment-based infrastructure for social relationships}

We have claimed that an agent-based framework should satisfy two requirements: 1) Explicit representation of the social relationship; 2) Social relationships should be first-class objects, which can be used for programming the agent behavior. 2COMM fulfills both requirements. Thanks to the social relationship abstraction, $2 \mathrm{COMM}$ enables an approach to agent programming that is not coupled to the chosen agent platform.

Currently, 2COMM supports social relationship-based agent programming for JADE and JaCaMo agents. JADE supplies standard agent services, i.e. message passing, distributed containers, naming and yellow pages services, agent mobility. When needed, an agent can enact a protocol role, which provides a set of operations by means of which agents participate in a mediated interaction session. JaCaMo [10] is a platform integrating Jason (as an agent programming language), CArtAgO and Moise (as a support to the realization of organizations). Normative/organizational specification is expressed as a Moise organization and translated into artifacts, that agents can decide to use.

We realize commitment-based interaction protocols by means of CArtAgO [21] artifacts. The core of $2 \mathrm{COMM}$ is in charge of management, maintenance and update of the social interaction state associated to each instance of a protocol artifact. CArtAgO provides a way to define and organize workspaces, that are logical groups of artifacts, that can be joined by agents at runtime. The environment is itself programmable and encapsulates services and functionalities. An API allows programming artifacts, regardless of the agent programming language or the agent framework used. This is possible by means of the agent body metaphor: CArtAgO provides a native agent entity, which allows using the framework as a complete MAS platform as well as it allows mapping the agents of some platform onto the CArtAgO agents, which, in this way, becomes a kind of "proxy" in the artifacts workspace. The former agent is the mind, that uses the CArtAgO agent as a body, interacting with artifacts. An agent interacts with an artifact by means of public operations, which can be equipped with guards: conditions that must hold in order for operations to produce their effects.

2COMM is organized as follows. Protocol roles are provided by communication artifacts, that are implemented by means of CArtAgO. Each communication artifact corresponds to a specific protocol enactment and maintains an own social state and an own communication state. Roles are linked to agents of the specific platform, via connector classes that implements the IPlayer interface. Figure 1 reports an excerpt of the 2 COMM UML class diagram ${ }^{1}$. Let us get into the depths of the implementation:

\footnotetext{
1 The source files of the system and examples are available at the URL http://di. unito.it/2COMM.
} 


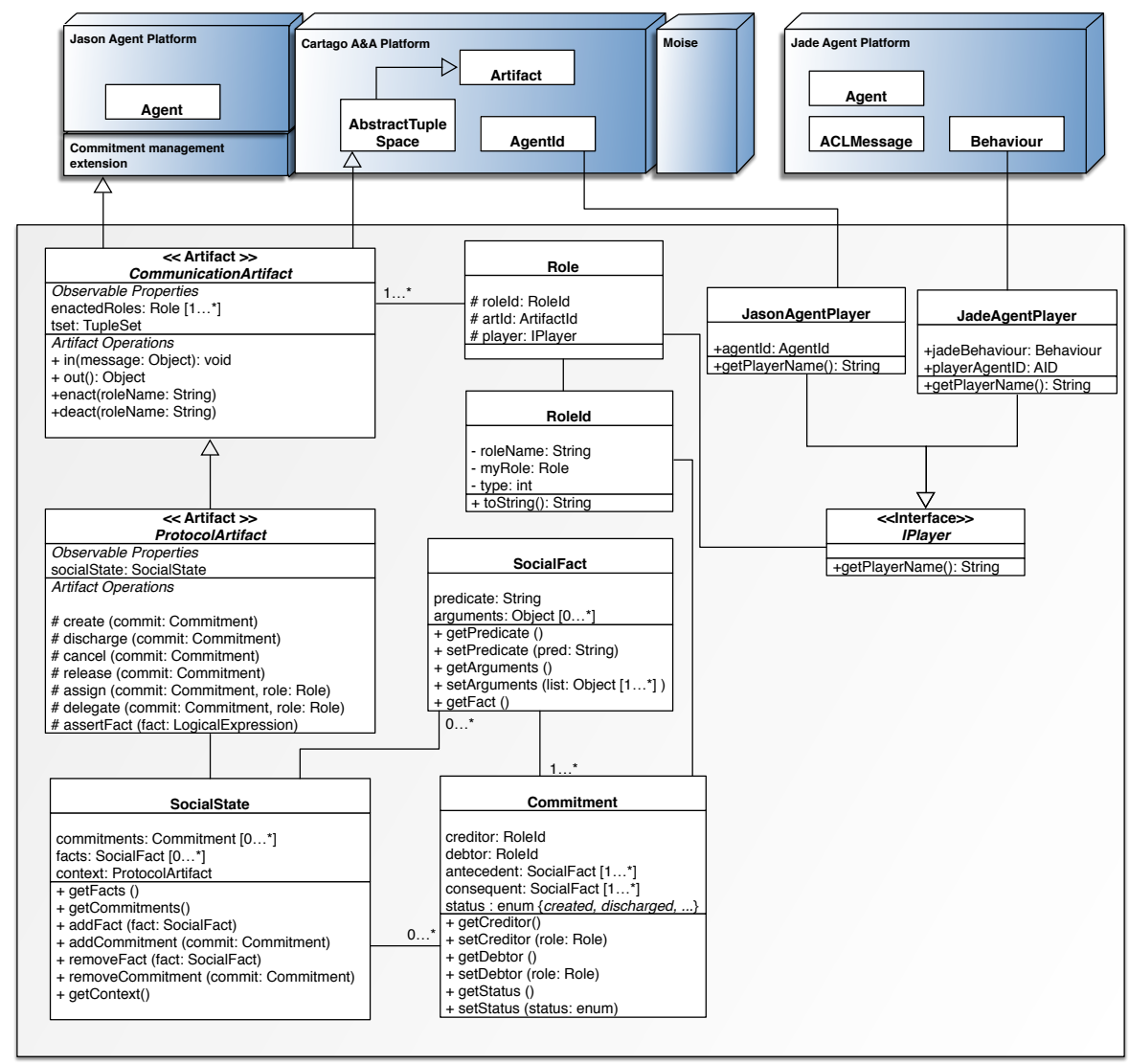

Fig. 1: Excerpt of the UML class diagram of 2COMM and connectors for JADE and JaCaMo.

- CommunicationArtifact (CA for short) provides the basic communication operations in and out for allowing mediated communication. CA extends an abstract version of the TupleSpace CArtAgO artifact: briefly, a blackboard that agents use as a tuple-based coordination means. In and out are, then, operations on the tuple space. CA also traces who is playing which role by using the property enactedRoles.

- Class Role extends the CArtAgO class Agent, and contains the basic manipulation logic of CArtAgO artifacts. Thus, any specific role, extending this super-type, will be able to perform operations on artifacts, whenever its player will decide to do so. Role provides static methods for creating artifacts and for enacting/deacting roles; the connector is in charge of linking agent and protocol through an instance of requested role. 
- The class CARole is an inner class of CA and extends the Role class. It provides the send and receive primitives, implemented based on the in and out primitives provided by $\mathrm{CA}$, by which agents can exchange messages.

- ProtocolArtifact (PA for short) extends CA and allows modeling the social layer with the help of commitments. It maintains the state of the ongoing protocol interaction, via the property socialState, a store of social facts and commitments, that is managed only by its container artifact. This artifact implements the operations needed to manage commitments (create, discharge, cancel, release, assign, delegate). PA realizes the commitment lifecycle and for the assertion/retraction of facts. Operations on commitments are realized as internal operations, that is, they are not invokable directly: the protocol social actions will use them as primitives to modify the social state. We refer to modifications occurred to the social state as social events. Being an extension of CA, PA maintains two levels of interaction: the social one (by commitments), and the communication one (by message exchange).

- The class PARole is an inner class of PA and extends the CARole class. It provides the primitives for querying the social state, e.g. for asking the commitments in which a certain agent is involved, and the primitives that allow an agent to become, through its role, an observer of the events occurring in the social state. For example, an agent can query the social state to verify if it contains a commitment with a specific condition as consequent, via the method existsCommitmentWithConsequent (InteractionStateElement el). Alternatively, an agent can be notified about the occurrence of a social event, provided that it implements the inner interface ProtocolObserver. Afterwards, it can start observing the social state. PARole also inherits the communication primitives defined in CARole.

- The class SocialFact represents a fact of some relevance for the ongoing interaction, that holds in the current state of interaction. A social fact is asserted for tracking the execution of a protocol action. Actions can have additional effects on the social state; in this case, corresponding social facts are added to it.

- The class IPlayer is the interface between roles and players adopting them. Currently 2COMM provides implementations for Jade (JadeBehaviourPlayer) and Jason (JasonAgentPlayer).

In order to specify a commitment-based interaction protocol, it is necessary to extend PA by defining the proper social and communicative actions as operations on the artifact itself. Since we want agents to act on artifacts only through their respective roles, when defining a protocol it is also necessary to create the roles. We do so by creating as many extensions of PARole as protocol roles. These extensions are realized as inner classes of the protocol: each such class will specify, as methods, the powers of a role. Powers allow agents who play roles to actually execute artifact operations. The typical schema will be:

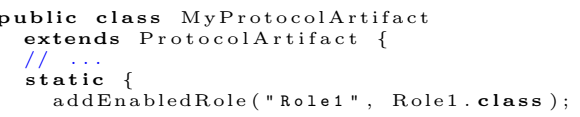




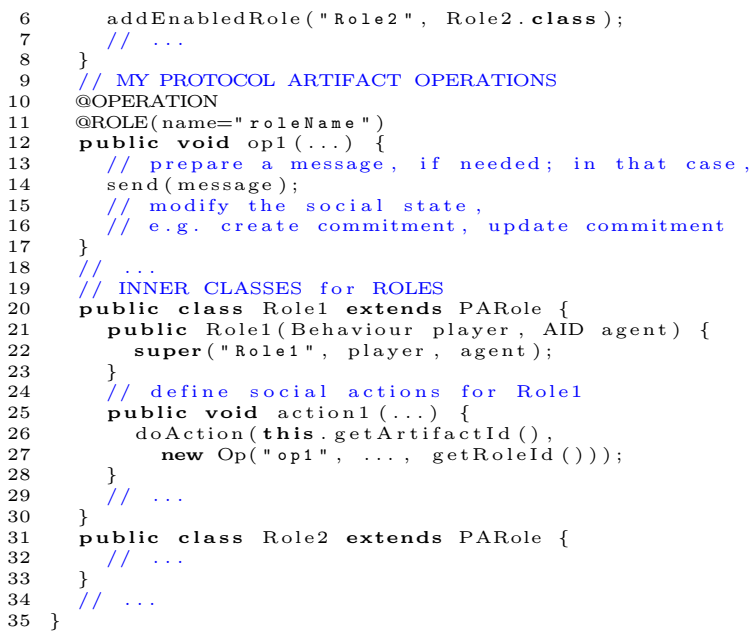

Protocol designers program the interaction protocol once. The resulting artifact can, then, be used in a JADE or in a JaCaMo context.

\subsection{Agent programming with 2COMM}

Agent programming with 2COMM amounts, at its core, to realizing a classical "sense-plan-act cycle", whose phases can be renamed "behold the social state", "activate behaviors according to social events", "schedule behavior execution". Beholding the social state, in 2COMM, does not require the agent to proactively sense it because agents can register to the protocol artifacts they use, and be notified by such artifacts of the occurrence of all events that are socially relevant. Agent programmers need to implement behaviors for those social events (e.g. commitment creation, commitment detachment) their agents are expected to handle. The occurrence of a social event for which an agent has a behavior to execute causes the activation of that behavior that will, then, be scheduled for execution by the agent platform. This mechanism represents an agent-oriented declination of callbacks. The agent paradigm forbids to use pure method invocation on the agent, that is autonomous by definition. Instead, the agent designer provides a collection of behaviours in charge of handling the different, possible evolutions of the social state, that are scheduled for execution when the corresponding conditions occur. For instance, when an agent has a behavior whose execution can create a commitment (the agent will be the debtor of that commitment), it needs to be able to tackle all events involved in the life cycle of that commitment (e.g. its detachment, satisfaction, violation, etc.). Instead, when, along the protocol execution, a conditional commitment may be created, whose creditor is the agent being implemented, then, a behavior that causes the detachment of the commitment should be included in the agent implementation. Behaviors for tackling the satisfaction/violation should also be considered. In this way, an intuitive programming guideline, based on social events, is provided to agent developers. 


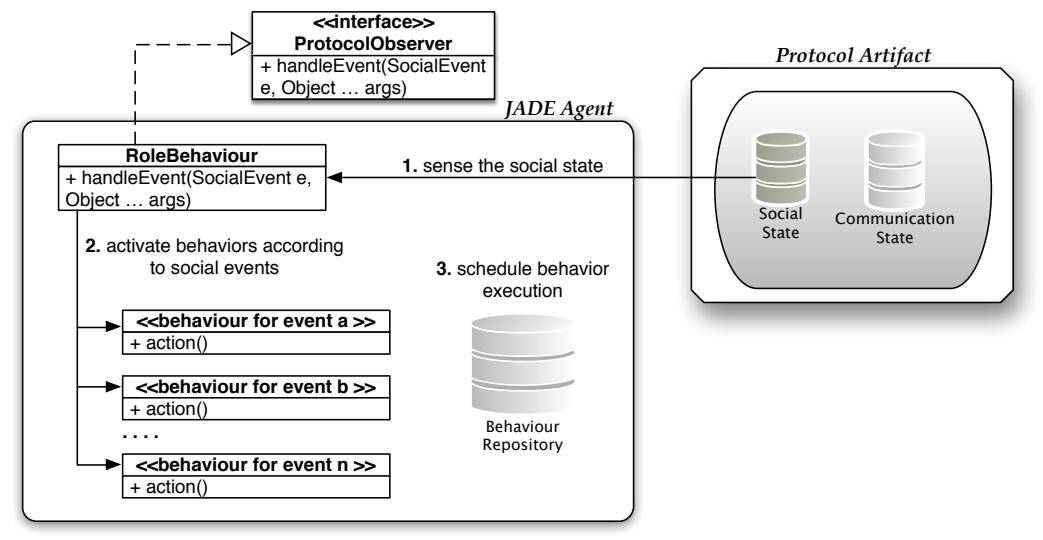

Fig. 2: 2COMM event handling schema for JADE agents.

Programming JADE agents with 2COMM. A JADE agent which plays a role in some protocol artifact needs to implement the method handleEvent in its behaviors (Figure 2). When events occur, the protocol artifact notifies them to the agents focusing on it. These react to such events by way of handleEvent. The agent programmer needs just to implement the logic for handling them, usually by adding proper behaviour(s) to the agent's behaviour repository. The occurrence of an event activates a corresponding behavior, if any is defined. When scheduled, the behaviour will be executed, and the event handled. The following is the pseudo-code of an example implementation:

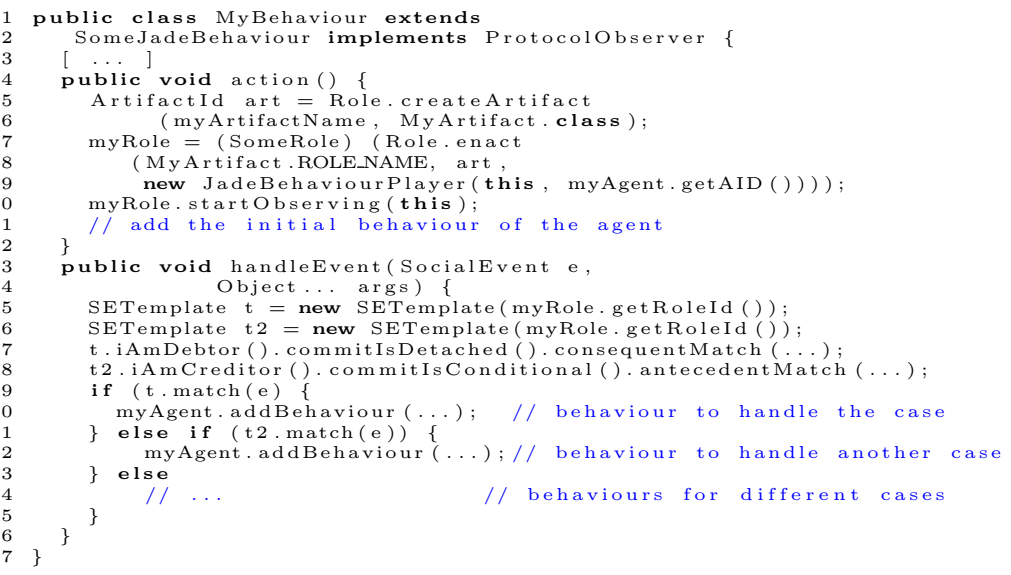

The basic schema, proposed for implementing a JADE behaviour, tracks how to handle social events that a protocol artifact notifies to an agent. Notification is performed through the handleEvent method, whose parameter contains the social effects of the event (e.g. if a commitment is added or satisfied, if a social 
fact is asserted, and such like). The implementation of handleEvent should contain conditions related to the occurred event. In JADE, event-related behaviours are added to the agent's behaviour library when a certain condition holds. If the social event to be notified is a commitment, it is possible to further check specific conditions of interest on it, including its state, the identity of its debtor and/or creditor, the antecedent or consequent condition (lines 19-22). The agent will, then, add appropriate behaviours to handle the detected situation. A templatebased matching mechanism for social events is provided (class SETemplate, lines 15-18) used by programmer in order to specify matching conditions. Each template class method returns this, thus compacting the code for construction of complex conditions simply using the standard method dot notation.

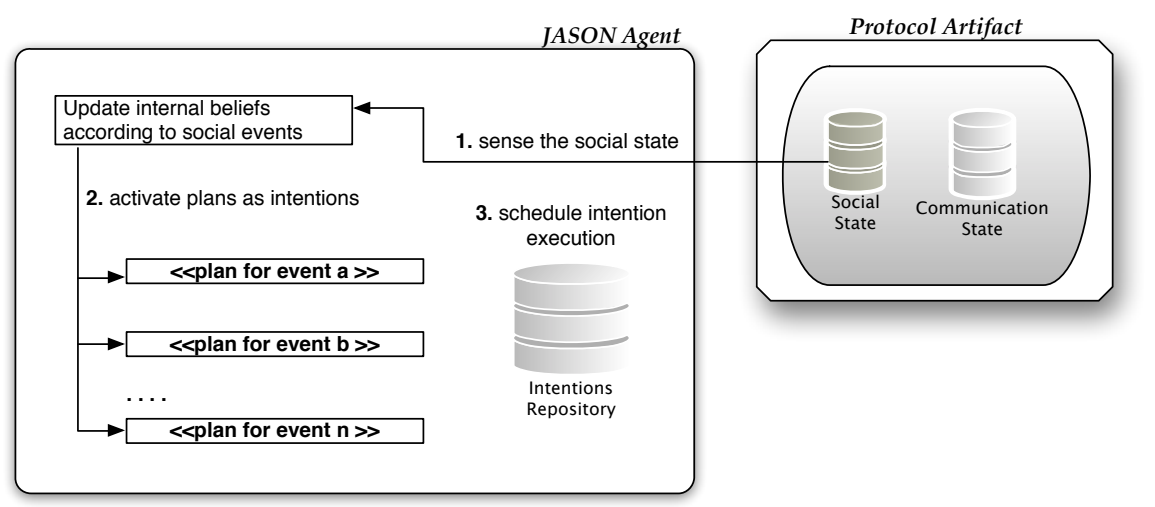

Fig. 3: 2COMM event handling schema for JaCaMo/Jason agents.

Programming JaCaMo agents with 2COMM. JaCaMo agents are programmed as Jason agents. A Jason agent has the capability of performing reasoning cycles, that is, the agent architecture performs a cycle of sense-plan-act, that allows agents to evaluate which plans are triggered for execution each time an event occurs. In this framework, social events can be modeled as regular Jason events, fired by the protocol artifact, thus, it is not required to the Jason agents to perform any processing of them. This is a difference with JADE, which, instead, provides the abstraction of agent only as a set of behaviours with communication capabilities. The adoption of artifacts that notify the occurrence of social events to focusing agents allows plan specifications whose triggering events involve social events (e.g. commitment creation), as depicted in figure 3. Social events can also be used inside a plan context or body. As a difference with beliefs, commitment assertion/deletion can only occur through the artifact, as a 
consequence of a modification of the social state. For example, this is the case that deals with commitment addition:

$+c c($ debtor, creditor, antecedent, consequent, status) :

$\langle$ context $\rangle \leftarrow\langle$ body $\rangle$.

The plan is triggered when a commitment that unifies with the plan head appears in the social state with the specified status. The syntax is the standard for Jason plans. Debtor and Creditor are to be substituted by the proper role names. The plan may be devised so as to achieve a change of the status of the commitment (e.g.: the debtor will satisfy the consequent, the creditor will satisfy the antecedent and so detach the commitment) or it may be devised to allow the agent to do something as a reaction (e.g. collecting information). A similar schema can be used in the case of commitment deletion and in the case of addition (deletion) of social facts. Commitments can also be used in contexts and in plans as test goals $(? c c(\ldots))$ or achievement goals $(! c c(\ldots))$. Addition or deletion of such goals can, as well, be managed by plans. For example:

$+!$ cc(debtor, creditor, antecedent, consequent, status) :

$\langle$ context $\rangle \leftarrow\langle$ body $\rangle$.

The plan is triggered when the agent creates an achievement goal concerning a commitment. Consequently, the agent will act upon the artifact so as to create the desired social relationship. After the execution of the plan the commitment cc(debtor, creditor, antecedent, consequent, status) will hold in the social state and will have been projected onto the belief bases of each of the agents which focused on the artifact.

\section{Programming Agents on Social Relationships: an example}

2COMM protocols constitute an outline for building agents that entails a uniform implementation of agent social abilities among different platforms: how to react to social events. We present a real implementation of the Contract Net Protocol (CNP) [17], and how JADE and Jason agents can be implemented. We adopt the following commitment-based CNP formulation:

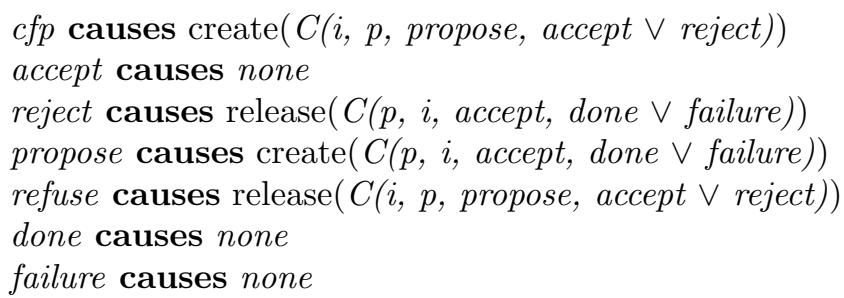

where $i$ stands for the role Initiator and $p$ for Participant. The execution of each such action has a social effect amounting to make the commitments which involve it progress, e.g. propose detaches $C(i, p$, propose, accept $\vee$ reject $)$. Additionally, they may cause further social effects which are those specified after 
"causes" (none means that the action execution has no further social effect but its occurrence). Initiator supplies its player the actions $c f p$ (call for proposal), accept, and reject. The first allows the initiator to ask participants for proposals for solving a task of interest. If a proposal is chosen, action accept notifies the winner and all other proposals are rejected. The role participant supplies its player the actions propose, refuse, done, and failure. Action propose allows a participant to supply a solution for a task, action refuse allows declining the invitation to send a proposal. If a proposal is accepted, the winning participant is expected to execute the task and either provide the result by means of the action done or communicate its failure. Actions affect the social state, e.g., when an Initiator executes $c f p$, the commitment $C(i, p$, propose, accept $\vee$ reject $)$ is added to the social state. This binds $i$ to either accept or reject a proposal, if one is received.

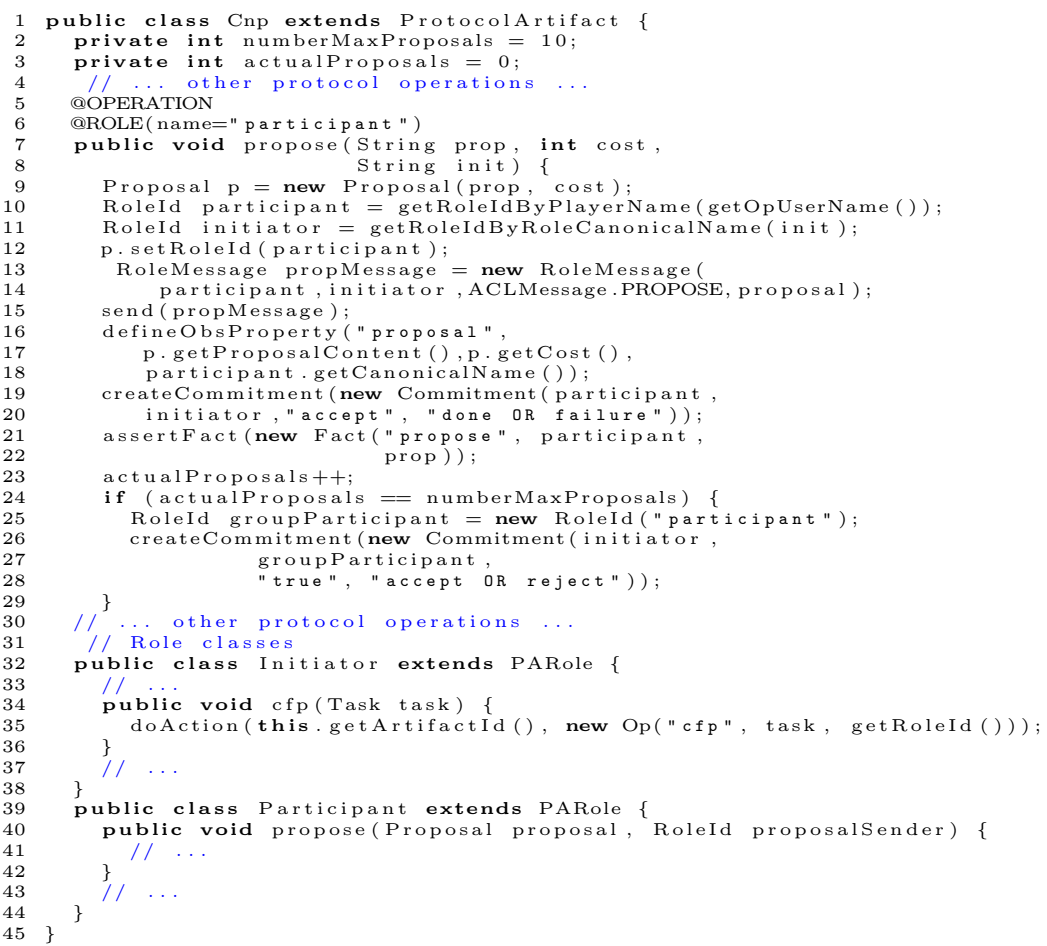

propose (line 7) is a social action. It is realized as a CArtAgO operation, in fact it is decorated by the CArtAgO Java annotation @OPERATION, line 5. It can be executed only by an agent playing the role participant. This is specified by the 2COMM Java annotation @ROLE(name="participant"), line 6. It asserts social fact (line 21), that traces the proposal made by the participant; then, it counts the received proposals and, when their number is sufficient, signals this fact to the initiator by the creation of a commitment (line 19) towards the group of participants. A message of performative PROPOSE (line 13) containing the participant's proposal is sent to the initiator. 
The proposed CNP implementation remains the same independently from the fact that it is used by a JADE or a Jason agents. 2COMM current version uses role internal classes for JADE agents, while these are ignored if the enacting agents are written in Jason. Let us now compare agents implementations to highlight similarities and analogies. We will focus on the code for the Initiator role, starting from a JADE agent.

JADE agents. Protocol designers can provide full support to JADE developers by implementing social-event adapters (see the listing below). So, agent developers will only need to implement the specific behaviours that tackle the events that are notified by the artifact. A clear advantage is an increased code reuse and modularization: the agent needs to be able to react to social events, adopting corresponding behaviours, and, therefore, the agent's autonomy is not jeopardized by extending the adapter. Here is a possible implementation for the Initiator adapter behaviour.

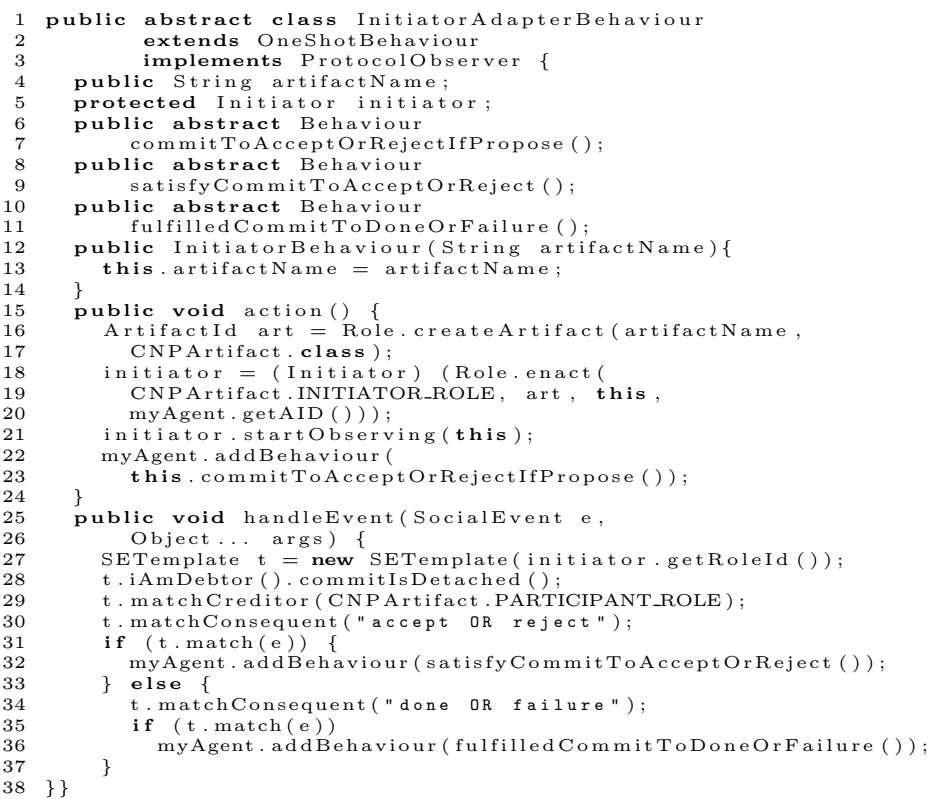

After line 21, all events, occurring in the social state, are notified to the role Initiator, which will handle them by executing handleEvent after a callback. The above abstract behaviour is extended by the concrete behaviour of the agent that plays the role Initiator. In particular, here we find the methods that create the actual behaviours for managing the social events.

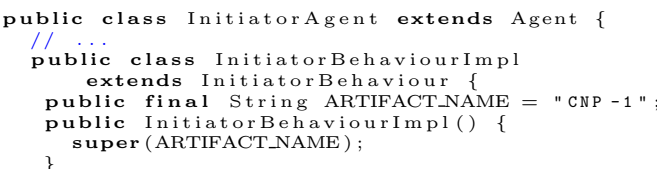




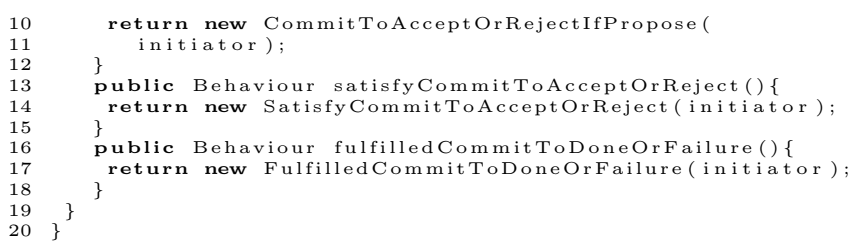

The agent logic is structured as a number of behaviours that are in charge of handling the social events. When a social event is received, the adapter loads the corresponding behaviour, that is scheduled for the execution. This is similar to how a Jason agent is programmed, that is, a collection of plans that become active when a trigger is satisfied. For example, we report the behaviour SatisfyCommitToAcceptOrReject, which gathers proposals and selects the one to accept $^{2}$.

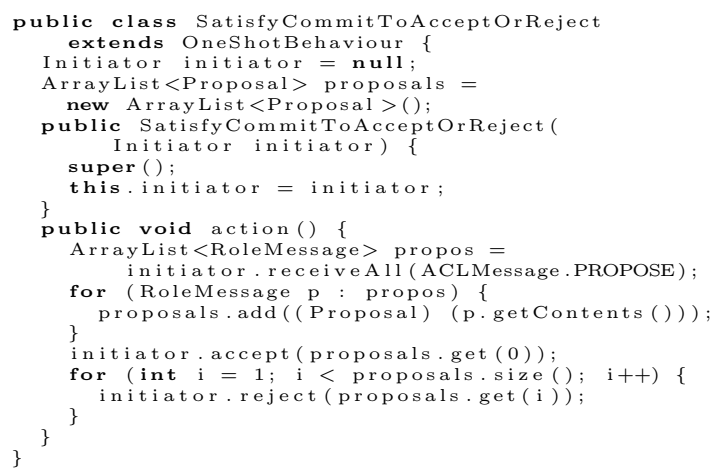

JaCaMo agents. The above implementation of SatisfyCommitToAcceptOrReject is analogous to how a Jason agent can be programmed to react to the same commitment:

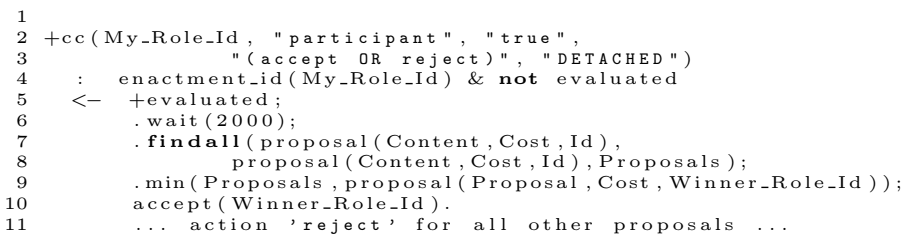

We now report and comment excerpts of Jason agent code for the Initiator.

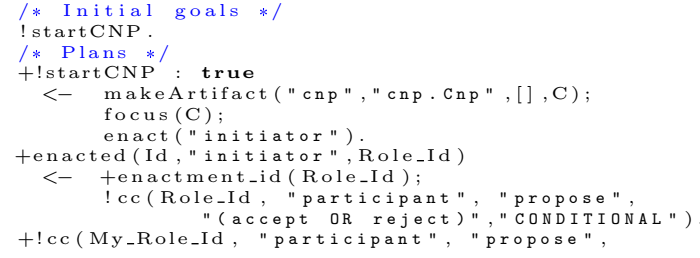

${ }^{2}$ The criterion here is trivial, the first proposal is the one which is accepted. 


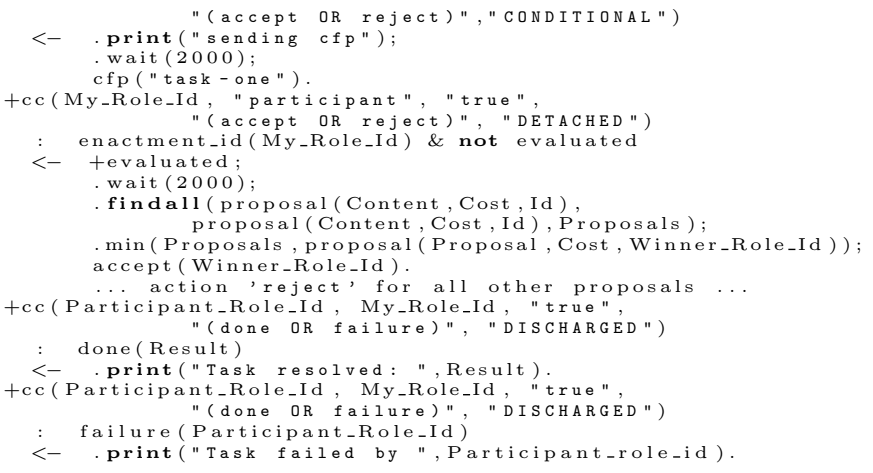

!startCNP, line 2 , is an initial goal, that is provided for beginning the interaction. In this implementation, the agent which plays the initiator role is in charge of creating the artifact (makeArtifact ("cnp", "cnp.Cnp",[],C)) that will be used for the interaction. The agent will, then, enact the role "initiator" (enact("initiator")); the artifact will notify the success of the operation by asserting an enacted belief. Since the program contains the plan triggered by the enacted belief, the initiator agent can, then, execute $c f p$. When enough participants will have committed to perform the task, in case their proposal is accepted ( $c c\left(M y_{-}\right.$Role_Id, "participant", "true", "(accept OR reject", "DETACHED"), the initiator agent evaluates the proposals and decides which to accept (we omit the reject case for sake of brevity).

\begin{tabular}{l|c|c|c|c} 
& JADE & + 2COMM & JaCaMo & + 2COMM \\
\hline Programmable communication channels & X & $\checkmark$ & $\checkmark$ & $\checkmark$ \\
Notification of social relationships of interaction & X & $\checkmark$ & X & $\checkmark$ \\
Interaction/agent logic decoupling & X & $\checkmark$ & X & $\checkmark$ \\
Expected behaviours reasoning & X & $\checkmark$ & $\checkmark$ & $\checkmark$ \\
Library of reusable patterns of interaction & $\checkmark$ & $\checkmark$ & X & $\checkmark$ \\
Runtime interaction monitoring & X & $\checkmark$ & $\checkmark$ & $\checkmark$ \\
Social-based Agent Programming Pattern & X & $\checkmark$ & X & $\checkmark$ \\
Norms and Obligations modeling & X & X & $\checkmark$ & $\checkmark$
\end{tabular}

Table 1: Comparison among JADE, JaCaMo and 2COMM improvements.

Remarks. Summarizing, a JADE agent leveraging 2COMM artifacts consists of a set of behaviours aimed at accomplishing given social relationships: such behaviours depend neither on when nor on how the social relationships of interest are created inside the social state. These aspects are, in fact, encoded in the protocol artifact that creates them based on the actions the agents perform. As a consequence, modifying how or when a social relationship is created does not have any impact on the agent implementation. Analogously for Jason agents, plans are not affected by modifications made on protocol: it is possible to adapt 
the interaction logic to different contexts without any impact on agents. Each plan is defined as reaction to a social event, whose evolution is stated by the artifact. Table 1 synthesizes a comparison among JADE and JaCaMo, highlighting aspects that are improved or added by 2COMM.

\section{Conclusions and Discussion}

In this work, we have proposed 2COMM, an infrastructure for allowing agents to interact by following an accepted set of regulations, with a self-governance approach. Self-governance mechanisms rely on the reification of commitments and of commitment-based protocols. These are, at all respects, resources that are made available to stakeholders and that are realized by means of artifacts. The proposal is characterized, on the one hand, by the flexibility and the openness that are typical of MAS, and, on the other, by the modularity and the compositionality that are typical requirements of the methodologies for design and development. One of the strong points of the proposal is the decoupling between the design of the agents and the design of the interaction, that builds on the decoupling between computation and coordination done by coordination models, like tuple spaces. This is a difference with respect to JADE or JaCaMo where no decoupling occurs: a pattern of interaction is projected into a set of JADE behaviours or Jason plans, one for each role. Binding the interaction to ad-hoc behaviours/plans does not allow having a global view of the protocol and complicates its maintenance.

2COMM supports programming JADE and Jason agents, by following a uniform approach. Moreover, agents programming can leverage the methodology

explained in [3]. It also simplifies the interaction of different agent platforms thanks to its connectors, which make protocol artifacts accessible and, thus, allow mediated communication between agents that belong to different platforms. This feature fulfills the purpose of supporting the development of heterogeneous and open agent systems. So, for instance, 2COMM enables the interaction of JADE agents with JaCaMo agents in a transparent and seamless way: it is not necessary to adapt an agent implementation to the platforms on which the other agents of the system run. For what concerns implementation, connectors bridge beteen CArtAgO and the used agent platforms. Any agent can take part to interaction sessions with others simply by using a protocol artifact. Note that Jason (which is part of JaCaMo) allows changing the communication infrastructure, switching to that of JADE, however, this choice is to be done a priori and has an impact on the design. 2COMM does not impose any choice a priori, guaranteeing the interaction between any pair of agent platforms for which a 2COMM connector exists.

2COMM complements the obligation-based specification of organizations, specifically suiting those situations where interaction is not subject to an organizational guideline, like in the case when interaction is among agents and each agent decides what is best for itself, or when guidelines amount to declarative, underspecified constraints that still leave agents the freedom to take strategic 
decisions on their behavior. In this case, interaction strongly relies on the two basic notions of goal and of engagement. For a thorough discussion of the differences between our proposal and organizational or normative approaches, please check [4].

Decoupling is an effect of explicitly representing social relationships as resources: agent behaviour is, thus, defined based on the existing social relationships and not on the process by which they are created. For instance, in CNP the initiator becomes active when the commitments that involve it as a debtor, and which bind it to accept or reject the proposals, are detached. It is not necessary to specify nor to manage, inside the agent, such things as deadlines or counting the received proposals: the artifact is in charge of these aspects. Testing 2COMM with Jason and JADE proved that programming agents starting from their desired interaction can be a valuable starting point, that can be extended towards a methodology useful for open and heterogeneous scenarios. We intend to explore this direction by adding connectors for different agent platforms.

Recently, we developed on top of 2COMM a commitment-based typing system [2] for JADE agents. Such typing includes a notion of compatibility, based on subtyping, which allows for the safe substitution of agents to roles along an interaction that is ruled by a commitment-based protocol. Type checking can be done dynamically when an agent enacts a role.

\section{References}

1. Matteo Baldoni, Cristina Baroglio, and Federico Capuzzimati. A commitmentbased infrastructure for programming socio-technical systems. ACM Transactions on Internet Technology (TOIT), 14(4):23, 2014.

2. Matteo Baldoni, Cristina Baroglio, and Federico Capuzzimati. Typing multi-agent systems via commitments. In Fabiano Dalpiaz, Jürgen Dix, and M.Birna van Riemsdijk, editors, Engineering Multi-Agent Systems, volume 8758 of Lecture Notes in Computer Science, pages 388-405. Springer International Publishing, 2014.

3. Matteo Baldoni, Cristina Baroglio, Federico Capuzzimati, and Roberto Micalizio. Empowering Agent Coordination with Social Engagement. In M. Gavanelli, E. Lamma, and F. Riguzzi, editors, $A I^{*} I A$ 2015: Advances in Artificial Intelligence, $X I V$ International Conference of the Italian Association for Artificial Intelligence, LNAI, Ferrara, Italy, September 2015. Springer. To appear.

4. Matteo Baldoni, Cristina Baroglio, Federico Capuzzimati, and Roberto Micalizio. Leveraging Commitments and Goals in Agent Interaction. In D. Ancona, M. Maratea, and V. Mascardi, editors, Proc. of XXX Italian Conference on Computational Logic, CILC 2015, Genova, Italy, July 2015.

5. Matteo Baldoni, Cristina Baroglio, Viviana Mascardi, Andrea Omicini, and Paolo Torroni. Agents, multi-agent systems and declarative programming: What, when, where, why, who, how? In 25 Years GULP, volume 6125 of Lecture Notes in Computer Science, pages 204-230. Springer, 2010.

6. Matteo Baldoni, Guido Boella, and Leendert van der Torre. Modelling the Interaction between Objects: Roles as Affordances. In J. Lang, F. Lin, and J. Wang, editors, Knowledge Science, Engineering and Management: First International Conference, KSEM, volume 4092 of LNCS, pages 42-54, Guilin City, China, August 5-8 2006. Springer. 
7. Matteo Baldoni, Guido Boella, and Leendert van der Torre. Interaction between objects in powerjava. Journal of Object Technology, 6(2), 2007.

8. F. Bellifemine, F. Bergenti, G. Caire, and A. Poggi. JADE - A Java Agent Development Framework. In Multi-Agent Programming: Languages, Platforms and Applications, volume 15 of Multiagent Systems, Artificial Societies, and Simulated Organizations, pages 125-147. Springer, 2005.

9. Guido Boella and Leendert W. N. van der Torre. The ontological properties of social roles in multi-agent systems: definitional dependence, powers and roles playing roles. Artificial Intelligence and Law, 15(3):201-221, 2007.

10. Olivier Boissier, Rafael H. Bordini, Jomi F. Hübner, Alessandro Ricci, and Andrea Santi. Multi-agent oriented programming with JaCaMo. Science of Computer Programming, 78(6):747 - 761, 2013.

11. Rafael H. Bordini, Lars Braubach, Mehdi Dastani, Amal El Fallah-Seghrouchni, Jorge J. Gómez-Sanz, João Leite, Gregory M. P. O'Hare, Alexander Pokahr, and Alessandro Ricci. A survey of programming languages and platforms for multiagent systems. Informatica (Slovenia), 30(1):33-44, 2006.

12. Frances M. T. Brazier, Barbara M. Dunin-Keplicz, Nick R. Jennings, and Jan Treur. Desire: Modelling Multi-Agent Systems in a Compositional Formal Framework. International Journal of Cooperative Information Systems, 06(01):67-94, March 1997.

13. Amit K. Chopra. Commitment Alignment: Semantics, Patterns, and Decision Procedures for Distributed Computing. PhD thesis, North Carolina State University, Raleigh, NC, 2009.

14. Amit K. Chopra and Munindar P. Singh. An Architecture for Multiagent Systems: An Approach Based on Commitments. In Proc. of ProMAS, 2009.

15. Rosaria Conte, Cristiano Castelfranchi, and Frank Dignum. Autonomous norm acceptance. In Intelligent Agents V, Agent Theories, Architectures, and Languages, ATAL '98, volume 1555 of Lecture Notes in Computer Science, pages 99-112. Springer, 1998.

16. Michael Fisher, Rafael H. Bordini, Benjamin Hirsch, and Paolo Torroni. Computational logics and agents: A road map of current technologies and future trends. Computational Intelligence, 23(1):61-91, 2007.

17. Foundation for Intelligent Physical Agents. FIPA Specifications, 2002. http://www.fipa.org.

18. Viviana Mascardi, Maurizio Martelli, and Leon Sterling. Logic-based specification languages for intelligent software agents. TPLP, 4(4):429-494, 2004.

19. Andrea Omicini, Alessandro Ricci, and Mirko Viroli. Artifacts in the A\&A metamodel for multi-agent systems. Autonomous Agents and Multi-Agent Systems, 17(3):432-456, 2008.

20. Andrea Omicini and Franco Zambonelli. TuCSoN: a coordination model for mobile information agents. In 1st International Workshop on Innovative Internet Information Systems (IIIS'98), pages 177-187. IDI - NTNU, Trondheim (Norway), 8-9 June 1998.

21. Alessandro Ricci, Michele Piunti, and Mirko Viroli. Environment programming in multi-agent systems: an artifact-based perspective. Autonomous Agents and Multi-Agent Systems, 23(2):158-192, 2011.

22. Munindar P. Singh. An ontology for commitments in multiagent systems. Artif. Intell. Law, 7(1):97-113, 1999.

23. Munindar P. Singh. A social semantics for agent communication languages. In Issues in Agent Communication, volume 1916 of LNCS, pages 31-45. Springer, 2000. 
24. Danny Weyns, Andrea Omicini, and James Odell. Environment as a first class abstraction in multiagent systems. Autonomous Agents and Multi-Agent Systems, 14(1):5-30, 2007.

25. Pinar Yolum and Munindar P. Singh. Commitment Machines. In Intelligent Agents VIII, 8th International Workshop, ATAL 2001, volume 2333 of LNCS, pages 235247. Springer, 2002. 